\title{
COMPOSIÇÃO DA MASTOFAUNA DE MÉdIO E GRANDE PORTE NA RESERVA INDÍGENA "PARABUBURE", MATO GROSSO, BRASIL"
}

\author{
Ednaldo Cândido Rocha ${ }^{2}$ e Elias Silva ${ }^{3}$
}

\begin{abstract}
RESUMO - O Cerrado, segundo maior bioma brasileiro, foi considerado um dos 25 hotspots do planeta, por apresentar alto grau de endemismo e ser uma das regiões biologicamente mais ricas e ameaçadas em termos mundiais. Mas muitas áreas desse bioma ainda carecem de conhecimentos elementares sobre seu potencial biológico, evidenciando-se a necessidade de gerar informações através de inventários e diagnósticos ambientais. Nesses termos, este estudo objetivou inventariar as espécies de mamíferos de médio e grande portes na Reserva Indígena "Parabubure", Município de Campinápolis, leste do Estado de Mato Grosso. Para tanto, no período compreendido entre 12 de agosto e 27 de setembro de 2005, a porção leste da reserva, com cerca de 25.000 ha, foi aleatoriamente percorrida em busca de evidências de mamíferos de médio e grande portes. A amostragem incluiu métodos diretos (sinais acústicos e visualizações) e indiretos (pegadas, fezes e tocas) para o registro das espécies. Foram obtidos registros de 30 espécies de mamíferos silvestres, 28 de médio e grande portes e duas de pequeno porte, sendo sete listadas como ameaçadas de extinção em nível nacional. Por fim, embora enfrente queimadas periódicas, aparentemente a principal fonte de ameaça à mastofauna na Reserva Indígena "Parabubure" é a caça, tanto a realizada pelos índios Xavantes, sem um plano de sustentabilidade baseado na capacidade de suporte de cada espécie, quanto a praticada por outros agentes.
\end{abstract}

Palavras-chave: Mamíferos, Cerrado e levantamento faunístico.

\section{COMPOSITION OF MEDIUM AND LARGE-SIZED MAMMALIAN FAUNA FROM “PARABUBURE” INDIAN RESERVE, MATO GROSSO STATE, BRAZIL}

\begin{abstract}
Cerrado, the second largest Brazilian biome, was considered as one of the 25 hotspots of the planet due to its high rate of endemism and because it is one the richest biological regions besides, also considered globally threatened. However, a lot of areas belonging to its biome are needed of elementary knowledge about its biological potential, highlighting the necessity of producing information by making inventories and environmental diagnostics. Based on that, this work aimed to create an inventory for the medium and large-sized mammalian species from the "Parabubure" Indian Reserve, Campinápolis county, Mato Grosso state east. Therefore, the East portion of the Reserve, rating around 25,000 ha, was randomly walked to search for medium and large-sized mammalian evidences during August $12^{\text {th }}$ to September $27^{\text {th }} 2005$. In order to register the species, the sampling included both direct (acoustics signs and visualizations) and indirect (track, feces and dens) methods. Thirty species of wild mammals, from which 28 were medium and largesized animals and 2 small-sized animals, were registered, seven of them happen to be listed as endangered species on national level. Lastly, in spite of the mammalian fauna from "Parabubure" Indian Reserve goes throw periodical burnt, apparently, the major threatening source to the mammalian fauna in the Indian Reserve is hunting, both practiced by Xavantes Indians, which has no sustainability plan based on each species capacity support, and other agents.
\end{abstract}

Keywords: Mammals, Cerrado and faunistical survey.

\footnotetext{
${ }^{1}$ Recebido em 25.09.2007 e aceito para publicação em 24.04.2009.

${ }^{2}$ Programa de Pós-Graduação em Ciência Florestal da Universidade Federal de Viçosa (UFV). E-mail: <ednaldorocha@ yahoo.com.br>.

${ }^{3}$ Departamento de Engenharia Florestal da UFV. E-mail: <eshamir@ufv.br>.
} 


\section{INTRODUÇÃO}

A influência das atividades humanas sobre espécies silvestres tem crescido numa razão sem precedentes. Neste sentido, análises mostram que a maior ameaça para as espécies de aves, mamíferos e anfíbios é a descaracterização e a degradação de seus habitats, impulsionadas pela agricultura e atividade florestal (BAILLIE et al., 2004).

O Cerrado constitui-se na segunda maior formação vegetal brasileira, ocupando cerca de 2 milhões de km2, que representam aproximadamente $23 \%$ do território nacional, apresentando fisionomias vegetais que englobam formações florestais, savânicas e campestres (RIBEIRO e WALTER, 1998). Nos últimos anos, o Cerrado tem enfrentado rápida redução de sua cobertura vegetal original devido à expansão da fronteira agropecuária na região central do Brasil. Cerca de metade da cobertura original desse bioma já foi transformada em pastagens plantadas, culturas anuais e outros tipos de uso (KLINK e MACHADO, 2005).

O Cerrado foi considerado um dos 25 hotspots do planeta, por apresentar alto grau de endemismo e ser uma das regiões biologicamente mais ricas e ameaçadas em termos mundiais (MYERS et al., 2000). Os mamíferos com ocorrência nesse bioma totalizam cerca de 195 espécies, sendo que 18 destas são endêmicas (MMA, 1999) e 17 estão incluídas na lista nacional das espécies da fauna brasileira ameaçadas de extinção (MMA, 2003).

O grau de ameaça e a importância ecológica do grupo tornam evidente a necessidade de incluir informações sobre os mamíferos terrestres de médio e grande porte em inventários e diagnósticos ambientais (PARDINI et al., 2003). Neste sentido, inventariar a fauna e flora de um ecossistema é o primeiro passo para sua conservação. Sem um conhecimento mínimo sobre a distribuição e a abundância dos organismos que ocorrem em um determinado local é praticamente impossível desenvolver qualquer projeto de preservação (SANTOS, 2003).

Os primeiros registros sobre a mastofauna da região do Vale do Araguaia, leste do estado de Mato Grosso, foram realizados durante a expedição anglo-brasileira Xavantina-Cachimbo, no final da década de 1960 (PINE et al., 1970). Posteriormente, poucos trabalhos abordando a comunidade de mamíferos de médio e grande porte foram desenvolvidos nessa região (FRAGOSO et al., 2000; PRADA e MARINHO-FILHO, 2004; ROCHA e
DALPONTE, 2006; ROCHA et al., 2006). Para o município de Campinápolis - MT não há levantamentos precedentes sobre a mastofauna, sendo que na área da Reserva Indígena "Parabubure" foi desenvolvido apenas um estudo com uma população de Lycalopex vetulus (Lund, 1842) (ROCHA, 2006).

Nestes termos, este trabalho objetivou inventariar as espécies de mamíferos de médio e grande porte na Reserva Indígena "Parabubure", município de Campinápolis, leste do estado de Mato Grosso. Os resultados apresentados neste estudo fornecem as primeiras informações sobre a comunidade de mamíferos no município de Campinápolis - MT e poderão contribuir para futuros planos de manejo e conservação da mastofauna da região.

\section{MATERIAL E MÉTODOS}

\section{1. Área de estudo}

Este estudo foi conduzido na Reserva Indígena "Parabubure" (224.447 ha), no município de Campinápolis, leste do estado de Mato Grosso, centro-oeste do Brasil (Figura 1). A área amostrada se localiza na porção leste da Reserva, entre os rios Couto Magalhães e Felipe, cuja superfície abrange cerca de 25.000 ha (ponto central com as seguintes coordenadas geográficas: $14^{\circ} 17^{\text {, }}$ S e $\left.52^{\circ} 56^{\prime} \mathrm{W}\right)$. Em linha reta, essa área está localizada a cerca de $30 \mathrm{~km}$ da cidade de Campinápolis e, segundo informações de antigos moradores da região, em 1967, já se encontrava habitada por índios da etnia Xavante. Dentro da porção amostrada, existe apenas uma aldeia, com aproximadamente 130 pessoas. Mas, em toda a área da Reserva, a população era de 3.357 pessoas em 2003 (SEPLAN, 2006).

A área de estudo está inserida no bioma Cerrado, com clima do tipo Aw (tropical estacional), segundo a classificação de Köppen, sendo caracterizado por duas estações bem definidas, uma seca (de abril a setembro) e uma chuvosa (de outubro a março) (NIMER, 1989). A precipitação pluviométrica média anual é de $1.500 \mathrm{~mm}$, sendo que mais de $90 \%$ das chuvas ocorrem de outubro a março. Durante a estação seca, a umidade relativa é baixa e a evaporação alta, sendo que a precipitação pode ser zero em alguns meses (ALHO e MARTINS, 1995). As temperaturas médias oscilam em torno de $25,5^{\circ} \mathrm{C}$, sendo junho e julho os meses mais frios e setembro o mais quente (PINTO JUNIOR e ROSSETE, 2005). 


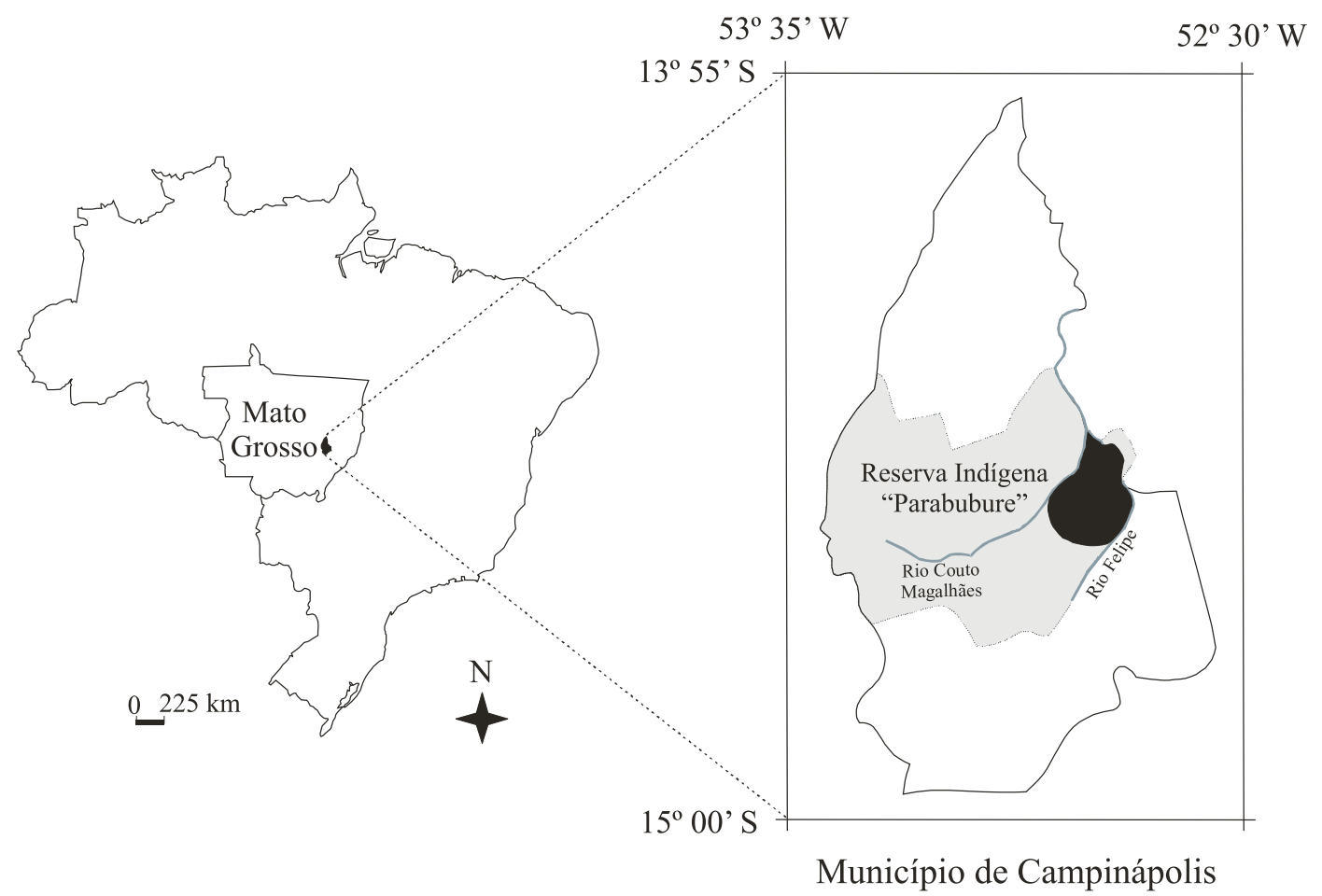

Figura 1 - Localização da Reserva Indígena "Parabubure”, em cinza, no município de Campinápolis - MT. A porção em preto representa a área amostrada.

Figure 1 - "Parabubure" Indian Reserve Localization is shown in gray, Campinápolis county, Mato Grosso state. The lot in black shows the area which was sampled.

O relevo da região é caracterizado como plano a ondulado, apresentando altitudes entre 250 e 300 $\mathrm{m}$ e com ocorrências de solos litólicos, cambissolo transicional com deposição de blocos de arenito e Latossolo Vermelho-Amarelo (RADAMBRASIL, 1981).

A porção amostrada da Reserva Indígena "Parabubure" possui fisionomias vegetais características do Bioma Cerrado, com o predomínio do campo sujo, tipo fitofisionômico exclusivamente herbáceo-arbustivo, com arbustos e subarbustos esparsos, incluindo uma parte cuja vegetação foi suprimida há cerca de 20 anos e que se encontra em estágio avançado de regeneração. Em menor escala, ocorrem também formações como floresta ripária, cerrado sensu stricto, campo limpo e campo de murundum.

$\mathrm{Na}$ área de estudo ocorrem distúrbios ocasionados por queimadas que a atingem total ou parcialmente. O fogo é utilizado, principalmente, durante os rituais de caça dos índios Xavantes e na limpeza e renovação de pastagens para um rebanho de aproximadamente 150 bovinos, criados em regime extensivo, e acaba se espalhando por extensas áreas.

\subsection{Coleta de dados}

A coleta de dados foi efetuada no período compreendido entre 12 de agosto e 27 de setembro de 2005, quando a área foi percorrida, à pé, à cavalo e de motocicleta, em busca de evidências de mamíferos de médio e grande porte. A amostragem incluiu métodos diretos (sinais acústicos e visualizações) e indiretos (pegadas, fezes e tocas) para o registro das espécies, sendo utilizados guias de campo para auxiliar na identificação dos animais (BECKER e DALPONTE, 1991; BORGES e TOMÁS, 2004). Esforço amostral diurno e noturno foi empregado para o inventário das espécies.

Levantamentos ao longo do dia foram conduzidos em todas as fisionomias vegetais presentes na área de estudo e obteve-se, principalmente, registros de pegadas e fezes, além de algumas visualizações de animais com atividade diurna. Métodos indiretos, especialmente 
a identificação de pegadas, têm sido amplamente utilizados em estudos sobre mamíferos de médio e grande porte, sobretudo para as espécies que são de difícil visualização em seus hábitats nativos (SCOSS et al., 2004; ROCHA e DALPONTE, 2006).

Levantamentos noturnos foram conduzidos, na área de campo sujo, em transectos dispostos ao longo de partes relativamente retilíneas de estradas e trilhas antigas, além de percursos lineares em meio à vegetação onde não havia caminhos. Os transectos (comprimento médio $=1,88 \mathrm{~km}$; desvio padrão $=0,99 \mathrm{~km}$; variação $=0,67$ a $4,70 \mathrm{~km}, \mathrm{n}=69$ levantamentos), estabelecidos de forma a cobrir a máxima porção da área de estudo, foram dispostos mantendo uma distância de $500 \mathrm{~m}$ entre eles, no caso de transecções paralelas amostradas na mesma noite.

A amostragem em transectos, distribuída em 22 noites de trabalho, totalizou $129,8 \mathrm{~km}$ percorridos durante caminhadas em dupla (numa velocidade média de 2,7 $\mathrm{km} / \mathrm{h}$ ), utilizando lanternas para visualizar os animais, binóculo para auxiliar na identificação e GPS para orientação linear ao longo do trajeto e para medir a distância total de cada transecto.

Neste estudo, foram considerados como mamíferos de médio e grande porte aqueles com peso corporal acima de $1 \mathrm{~kg}$ quando adultos, conforme sistema adotado por Chiarello (2000). Desta forma, embora Galea sp. e Sylvilagus brasiliensis (Linnaeus, 1758) sejam espécies de pequeno porte, foram incluídas neste estudo, pois puderam ser seguramente identificadas na área amostrada. O peso dos animais foi consultado em Fonseca et al. (1996).

\section{RESULTADOS E DISCUSSÃO}

Foram obtidos registros de 30 espécies de mamíferos silvestres, sendo 28 de médio e grande porte e duas de pequeno porte, distribuídas em nove Ordens (Tabela 1), conforme a classificação taxonômica de Wilson e Reeder (2005). Resultado relativamente parecido foi obtido por Rocha e Dalponte (2006), no município vizinho (Nova Xavantina), onde houve registro de 29 espécies de mamíferos numa reserva de Cerrado.

Tabela 1 - Taxa, nome comum e tipo de registro de mamíferos encontrados na Reserva Indígena "Parabubure", em Campinápolis - MT

Table 1 -Rate, common name and type of register for mammals found on the "Parabubure" Indian Reserve, Campinápolis, Mato Grosso state

\begin{tabular}{|c|c|c|c|}
\hline Taxa & Nome comum & Registro & Ambiente \\
\hline \multicolumn{4}{|l|}{ Ordem Didelphimorphia } \\
\hline \multicolumn{4}{|l|}{ Família Didelphidae } \\
\hline Didelphis albiventris Lund, 1840 & gambá, mucura & visualização e pegadas & campo sujo e floresta ripária \\
\hline \multicolumn{4}{|l|}{ Ordem Pilosa } \\
\hline \multicolumn{4}{|l|}{ Família Myrmecophagidae } \\
\hline Myrmecophaga tridactyla Linnaeus, 1758* & tamanduá-bandeira & pegadas & campo sujo \\
\hline Tamandua tetradactyla (Linnaeus, 1758) & tamanduá-mirim & visualização e pegadas & campo sujo e cerrado sensu stricto \\
\hline \multicolumn{4}{|l|}{ Ordem Cingulata } \\
\hline \multicolumn{4}{|l|}{ Família Dasypodidae } \\
\hline Dasypus novemcinctus Linnaeus, 1758 & tatu-galinha & pegadas & campo sujo \\
\hline Dasypus septemcinctus Linnaeus, 1758 & tatu-mulita, tatuí & restos mortais & campo sujo \\
\hline $\begin{array}{l}\text { Euphractus sexcinctus (Linnaeus, 1758) } \\
\text { Priodontes maximus (Kerr, 1792)* }\end{array}$ & $\begin{array}{l}\text { tatu-peba, tatu-peludo } \\
\text { tatu-canastra }\end{array}$ & $\begin{array}{l}\text { pegadas e tocas } \\
\text { tocas }\end{array}$ & $\begin{array}{l}\text { campo sujo e cerrado sensu stricto } \\
\text { campo sujo }\end{array}$ \\
\hline \multicolumn{4}{|l|}{ Ordem Primates } \\
\hline \multicolumn{4}{|l|}{ Família Cebidae } \\
\hline Cebus libidinosus (Spix, 1823) & macaco-prego & $\begin{array}{l}\text { visualização e } \\
\text { vocalização }\end{array}$ & floresta ripária \\
\hline \multicolumn{4}{|l|}{ Família Atelidae } \\
\hline Alouatta caraya (Humboldt, 1812) & bugio, guariba & $\begin{array}{l}\text { visualização e } \\
\text { vocalização }\end{array}$ & floresta ripária \\
\hline
\end{tabular}

R. Árvore, Viçosa-MG, v.33, n.3, p.451-459, 2009 
Tabela 1 - Cont.

Table 1 - Cont.

\begin{tabular}{|c|c|c|c|}
\hline Taxa & Nome comum & Registro & Ambiente \\
\hline \multicolumn{4}{|l|}{ Ordem Carnivora } \\
\hline \multicolumn{4}{|l|}{ Família Canidae } \\
\hline Cerdocyon thous (Linnaeus, 1766) & cachorro-do-mato & pegadas e fezes & campo sujo e cerrado sensu stricto \\
\hline Chrysocyon brachyurus (Illiger, 1815)* & lobo-guará & $\begin{array}{l}\text { pegadas, fezes } \\
\text { e vocalização }\end{array}$ & campo sujo e cerrado sensu stricto \\
\hline Lycalopex vetulus (Lund, 1842) & raposa-do-campo & $\begin{array}{l}\text { visualização, } \\
\text { pegadas e fezes }\end{array}$ & campo sujo e cerrado sensu stricto \\
\hline \multicolumn{4}{|l|}{ Família Procyonidae } \\
\hline Procyon cancrivorus (G. Cuvier, 1798) & mão-pelada & pegadas & campo sujo \\
\hline \multicolumn{4}{|l|}{ Família Mustelidae } \\
\hline Eira barbara (Linnaeus, 1758) & irara, papa-mel & visualização e pegadas & campo sujo e cerrado sensu stricto \\
\hline \multicolumn{4}{|l|}{ Família Felidae } \\
\hline Puma concolor (Linnaeus, 1771)* & onça-parda, suçuarana & $\begin{array}{l}\text { pegadas e } \\
\text { restos mortais }\end{array}$ & campo sujo e floresta ripária \\
\hline Puma yagouaroundi (É. Geoffroy, 1803) & gato-mourisco & pegadas & campo sujo \\
\hline Leopardus pardalis (Linnaeus, 1758)* & jaguatirica & $\begin{array}{l}\text { pegadas e } \\
\text { restos mortais }\end{array}$ & campo sujo e floresta ripária \\
\hline Leopardus wiedii $(\mathrm{Schinz}, 1821)^{*}$ & gato-maracajá & restos mortais & floresta ripária \\
\hline Não identificada & gato-do-mato & pegadas & campo sujo \\
\hline \multicolumn{4}{|l|}{ Ordem Perissodactyla } \\
\hline Tapirus terrestris (Linnaeus, 1758) & anta & pegadas & campo sujo e floresta ripária \\
\hline \multicolumn{4}{|l|}{ Ordem Artiodactyla } \\
\hline \multicolumn{4}{|l|}{ Família Tayassuidae } \\
\hline Pecari tajacu (Linnaeus, 1758) & cateto, caititu & $\begin{array}{l}\text { pegadas e } \\
\text { restos mortais }\end{array}$ & campo sujo e floresta ripária \\
\hline Tayassu pecari (Link, 1795) & queixada & pegadas & floresta ripária \\
\hline \multicolumn{4}{|l|}{ Família Cervidae } \\
\hline Blastocerus dichotomus (Illiger, 1815)* & cervo-do-pantanal & pegadas & campo sujo \\
\hline Mazama gouazoubira (G. Fischer, 1814) & veado-catingueiro & visualização e pegadas & campo sujo \\
\hline Ozotoceros bezoarticus (Linnaeus, 1758) & veado-campeiro & visualização e pegadas & campos sujo e campo limpo \\
\hline \multicolumn{4}{|l|}{ Ordem Rodentia } \\
\hline \multicolumn{4}{|l|}{ Família Caviidae } \\
\hline Hydrochoerus hydrochaeris (Linnaeus, 1766) & capivara & pegadas e fezes & floresta ripária \\
\hline Galea sp. & preá & visualização & campo sujo e campo limpo \\
\hline \multicolumn{4}{|l|}{ Família Cuniculidae } \\
\hline Cuniculus paca (Linnaeus, 1766) & paca & visualização e pegadas & campo sujo e floresta ripária \\
\hline \multicolumn{4}{|l|}{ Família Dasyproctidae } \\
\hline Dasyprocta $\mathrm{sp}$ & cutia & visualização e pegadas & floresta ripária \\
\hline \multicolumn{4}{|l|}{ Ordem Lagomorpha } \\
\hline \multicolumn{4}{|l|}{ Família Leporidae } \\
\hline Sylvilagus brasiliensis (Linnaeus, 1758) & tapetí, coelho & pegadas e fezes & campo sujo \\
\hline
\end{tabular}

* Espécies ameaçadas de extinção (MMA, 2003).

* Threatened of extinction species (MMA, 2003).

As Ordens mais representativas foram Carnivora (33,33\%; 10 espécies) e Artiodactyla (16,68\%; 5 espécies), alcançando juntas a metade das espécies registradas; seguidas por Cingulata e Rodentia, ambas com (13,33\%; 4 espécies), e por Pilosa e Primates, as duas com (6,67\%; 2 espécies). Por fim, as menos representativas foram Didelphimorphia, Perissodactyla e Lagomorpha, cada uma delas com $(3,33 \% ; 1$ espécie $)$ das espécies registradas (Figura 2).

A lista apresentada neste estudo foi composta por espécies identificadas principalmente por suas pegadas diagnósticas e por visualizações. Adicionalmente, outros indícios, como vocalizações, tocas e fezes, também foram 
considerados, mas não se incluiu informações provenientes de entrevistas. A maior abundância de registros na área de campo sujo pode não estar relacionada à preferência das espécies por esse tipo de habitat e sim em função de ser a fitofisionomia predominante na área amostrada.

Dentre as espécies registradas, sete estão listadas como ameaçadas de extinção em nível nacional (MMA, 2003): Myrmecophaga tridactyla Linnaeus, 1758, Priodontes maximus (Kerr, 1792), Chrysocyon brachyurus (Illiger, 1815), Puma concolor (Linnaeus, 1771), Leopardus pardalis (Linnaeus, 1758), Leopardus wiedii (Schinz, 1821) e Blastocerus dichotomus (Illiger, 1815).

Houve registro, por pegadas, de apenas um indivíduo de $B$. dichotomus e, da mesma forma, foi identificado um pequeno grupo de Tayassu pecari (Link, 1795), aparentemente, com cerca de dez indivíduos. Na atualidade, essas espécies parecem ser raras na Reserva Indígena "Parabubure"; mas, segundo informações de antigos moradores locais, elas já foram comuns nessa área. Além disto, segundo eles, também foi notória a redução nas populações de outros cervídeos, especialmente de Ozotoceros bezoarticus (Linnaeus, 1758).

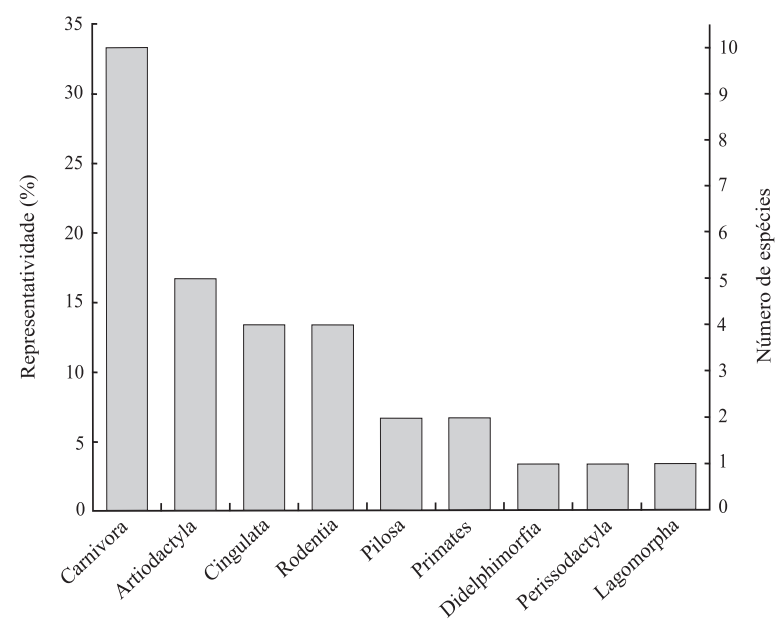

Figura 2 - Representatividade e número de espécies das Ordens de mamíferos de médio e grande porte registradas na Reserva Indígena "Parabubure", Campinápolis - MT.

Figure 2-Representability and number of species in the taxonomic orders of medium and large-sized mammalians registered on the "Parabubure" Indian Reserve, Campinápolis, Mato Grosso state.
Apesar de não terem sido registradas no presente estudo, é esperado que Nasua nasua (Linnaeus, 1766), Mazama americana (Erxleben, 1777), Panthera onca (Linnaeus, 1758) e Coendou prehensilis (Linnaeus, 1758) também ocorram na Reserva Indígena "Parabubure", mesmo que em baixas densidades, já que moradores locais atestam a existência dessas espécies na região. Além disto, há relatos que caçadores já tenham abatido $M$. americana e $P$. onca em sítios próximos à área amostrada.

A ocorrência de caça dentro dos limites da Reserva Indígena "Parabubure" é um importante fator de ameaça à fauna, na medida em que pode afetar a distribuição e a abundância das espécies. Neste sentido, Cullen Júnior et al. (2000), avaliando o efeito da caça sobre mamíferos em fragmentos de Mata de Planalto no estado de São Paulo, observaram que as espécies registradas apresentaram abundância, em média, 37\% menor em áreas com forte pressão de caça, quando comparadas com áreas submetidas à baixa pressão de caça. Ainda segundo esses autores, os mamíferos ungulados foram os que mostraram um efeito da caça mais marcante, com abundância $69 \%$ menor em áreas com forte pressão de caça. Lopes e Ferrari (2000) comentam que as espécies de mamíferos terrestres caçadas apresentam geralmente um importante declínio populacional no início do processo, especialmente onde a pressão de caça é intensa, que pode levar a extinções locais ao longo do tempo.

Além de ações individuais, os índios Xavantes também praticam a caça coletiva, incluindo-se a feita com o uso do fogo. As espécies de mamíferos mais abatidas são as de médio e grande porte, tais como as pertencentes às Ordens Pilosa (tamanduás), Cingulata (tatus), Perissodactyla (anta) e Artiodactyla (cervídeos, catetos e queixadas) (FRAGOSO et al., 2000).

Como se pôde notar através de observações diretas, a caça por não-Xavantes também ocorre na Reserva Indígena "Parabubure", principalmente por pessoas advindas das proximidades. Mas, há também a ação de madeireiros que atuam de forma ilegal, pois abatem animais e pescam de forma descontrolada. Dentre os animais caçados, Cuniculus paca (Linnaeus, 1766) parece ser a espécie mais apreciada, seguida por Tapirus terrestris (Linnaeus, 1758), Pecari tajacu (Linnaeus, 1758), Hydrochoerus hydrochaeris (Linnaeus, 1766) e cervídeos.

Outro fator importante a ser considerado é a

R. Árvore, Viçosa-MG, v.33, n.3, p.451-459, 2009 
ocorrência de queimadas, já que grandes incêndios podem trazer drásticas consequiências, de forma direta e indireta, para a mastofauna, causando inclusive a morte de indivíduos de grande porte, tais como de tamanduá-bandeira, veado-campeiro, anta e tatu-canastra (SILVEIRA, 1999).

Como as queimadas atingem periodicamente a área da Reserva Indígena "Parabubure", há pouco acúmulo de massa vegetal combustível, de forma que o fogo não alcança grandes proporções. Neste sentido, Prada e Marinho-Filho (2004) comentam que a tradicional caça com fogo empregada pelos índios Xavantes funciona como um mecanismo para a prevenção de incêndios destrutivos. Além disto, os cursos d'água e as florestas ripárias presentes na área devem funcionar como barreiras naturais contra o fogo. Assim, é provável que a morte de mamíferos de médio e grande porte pelo efeito direto do fogo seja atenuada por este fator, corroborando com o observado por Prada e Marinho-Filho (2004) na Reserva Xavante do Rio das Mortes.

Por outro lado, no período pós-fogo, certamente há redução na quantidade de alimento, abrigo e habitat disponível, causando indiretamente efeitos adversos sobre a comunidade de mamíferos. Neste sentido, Silveira (1999) comenta que o maior efeito negativo do fogo sobre os mamíferos carnívoros deve ocorrer posteriormente aos incêndios, quando as populações de presas caem a números baixos em decorrência da intensa predação devido à exposição aos predadores naturais, falta de alimento e supressão de seus habitats.

Por fim, os resultados deste estudo indicam que a Reserva Indígena "Parabubure" desempenha importante papel na conservação da mastofauna da região, na medida em que abriga considerável riqueza de espécies de mamíferos de médio e grande porte, com algumas de grande interesse conservacionista. Mas, a área carece de planos de manejo florestal e faunístico, em que neste último se poderia tratar de aspectos relacionados à superexploração, a fim de evitar a extinção local de espécies. Além disto, é altamente desejável a implantação de um sistema eficiente de fiscalização da caça, pesca e retirada de madeira dentro dos limites da Reserva.

\section{CONCLUSÕES}

Foram obtidos registros de 30 espécies de mamíferos na Reserva Indígena "Parabubure", sendo 28 de médio e grande porte e duas de pequeno porte, das quais sete estão listadas como ameaçadas de extinção em nível nacional.

É notória a necessidade de incrementar a fiscalização contra a ação de caçadores não-Xavantes que agem de forma ilegal dentro da Reserva.

Novos estudos de longa duração com a comunidade de mamíferos são recomendados para a Reserva Indígena "Paraburure", visando monitorar flutuações populacionais ao longo do tempo e entre as estações, além do estabelecimento de um plano de manejo faunístico, de forma a evitar extinção local de espécies.

\section{AGRADECIMENTOS}

Ao Sr. Claudemiro Gomes da Silva e à Sra. Maria Lúcia Alves da Silva, pela acolhida em sua residência e pelo inestimável auxílio durante toda a coleta de dados.

\section{REFERÊNCIAS}

ALHO, C. J. R.; MARTINS, E. S. (Eds.). De grão em grão, o Cerrado perde espaço: Cerrado - impactos do processo de ocupação. Brasília: WWF, Pró-Cer, 1995. 66p.

BAILLIE, J. E. M.; HILTON-TAYLOR, C.; STUART, S. N. (Eds.). 2004 IUCN red list of threatened species: a global species assessment. Cambridge: IUCN, 2004. 191p.

\section{BECKER, M.; DALPONTE, J. C. Rastros de} mamíferos silvestres brasileiros: um guia de campo. Brasília: Universidade de Brasília, 1991. 181p.

BORGES, P. A. L.; TOMÁS, W. M. Guia de rastros e outros vestígios de mamíferos do Pantanal. Corumbá: Embrapa Pantanal, 2004. 139p.

BRASIL - Ministério do Meio Ambiente. Ações prioritárias para a conservação da biodiversidade do Cerrado e Pantanal. Brasília: Funatura, Conservation International, Fundação Biodiversitas, Universidade de Brasília, 1999. 26p.

BRASIL - Ministério do Meio Ambiente. Espécies da fauna brasileira ameaçadas de extinção.

Instrução Normativa $n^{\circ} 3$, de 27 de maio de 2003. Diário Oficial da União. de 28 de maio de 2003, n 101, Seção 1. p.88-97.

R. Árvore, Viçosa-MG, v.33, n.3, p.451-459, 2009 
CHIARELlO, A. G. Density and population size of mammals in remnants of Brazilian Atlantic Forest. Conservation Biology, v.14, n.6, p.1649-1657, 2000.

CULLEN JÚNIOR, L.; BODMER, R. E.; VALLADARESPÁDUA, C. Effects of hunting in habitat fragments of the Atlantic Forests, Brazil. Biological Conservation, v.95, n.1, p.49-56, 2000.

FONSECA, G. A. B. et al. Lista anotada dos mamíferos do Brasil. Belo Horizonte: Conservation International \& Fundação Biodiversitas, 1996. 38p. (Occasional Papers, 4).

FRAGOSO, J. M. V.; SILVIUS, K. M.; PRADA, M. Manejo de fauna na Reserva Xavante Rio das Mortes: cultura indígena e método cientifico integrados para conservação. Brasília: WWF, 2000. v.4. 68p.

KLINK, C. A.; MACHADO, R. B. A conservação do Cerrado brasileiro. Megadiversidade, v.1, n.1, p.147-155, 2005.

LOPES, M. A.; FERRARI, S. F. Effects of Human Colonization on the abundance and diversity of mammals in eastern Brazilian Amazônia.

Conservation Biology, v.14, n.6, p.1658-1665, 2000.

MYERS, N. et al. Biodiversity hotspots for conservation priorities. Nature, v.403, n.24, p.853-858, 2000.

NIMER, E. Climatologia do Brasil. Rio de Janeiro: IBGE, 1989. 422p.

PARDINI, R. et al. Levantamento rápido de mamíferos terrestres de médio e grande porte. In: CULLEN JÚNIOR, L.; RUDRAN, R.;

VALlADARES-PADUA, C. (Orgs.). Métodos de estudos em biologia da conservação e manejo da vida silvestre. Curitiba: Universidade Federal do Paraná, 2003. p.181-201.

PINE, R. H.; BISHOP, I. R.; JACKSON, R. J. Preliminary list of mammals of the Xavantina/Cachimbo expedition (Central Brazil). Transactions of the Royal Society of Tropical Medicine and Hygiene, v.64, n.5, p.668-670, 1970.

PINTO JUNIOR, O. B.; ROSSETE, A. N. Caracterização morfométrica da bacia hidrográfica do ribeirão Cachoeira, MT-Brasil. Geoambiente on-line, n.4, p.38-53, 2005.

R. Árvore, Viçosa-MG, v.33, n.3, p.451-459, 2009
PRADA, M.; MARINHO-FILHO, J. Effects of fire on the abundance of Xenarthrans in Mato Grosso, Brazil. Austral Ecology, v.29, n.5, p.568-573, 2004.

RADAMBRASIL. Levantamento dos recursos naturais. Ministério das Minas e Energia, 25, Folha SD - 22/Goiás, 1981.

RIBEIRO, J. F.; WALTER, B. M. T. Fitofisionomias do bioma Cerrado. In: SANO, S. M.; ALMEIDA, S. P. (Eds.). Cerrado: ambiente e flora. Planaltina: Embrapa, 1998. p.89-166.

ROCHA, E. C. Aspectos da história natural e conservação de Pseudalopex vetulus (Lund, 1842) (Carnivora: Canidae). 2006. 67f. Dissertação (Mestrado em Ciência Florestal) - Universidade Federal de Viçosa, Viçosa, MG, 2006.

ROCHA, E. C.; DALPONTE, J. C. Composição e caracterização da fauna de mamíferos de médio e grande porte em uma pequena reserva de Cerrado em Mato Grosso, Brasil. Revista Árvore, v.30, n.4, p.669-678, 2006.

ROCHA, E. C. et al. Evaluación estacional de la riqueza y abundancia de especies de mamíferos en la Reserva Biológica Municipal "Mário Viana", Mato Grosso, Brasil. Revista de Biología Tropical (International Journal of Tropical Biology and Conservation), v.54, n.3, p.879-888, 2006.

SANTOS, A. J. Estimativas de riqueza em espécies. In: CULLEN JÚNIOR, L.; RUDRAN, R.; VALlADARES-PADUA, C. (Orgs.). Métodos de estudos em biologia da conservação e manejo da vida silvestre. Curitiba: Universidade Federal do Paraná, 2003. p.181-201.

SCOSS, L. M. et al. Uso de parcelas de areia para o monitoramento de impacto de estradas sobre a riqueza de espécies de mamíferos. Revista Árvore, v.28, n.1, p.121-127, 2004.

SEPLAN - Secretaria de Estado de Planejamento e Coordenação Geral. Áreas indígenas, extensão, etnia, população total, município e situação jurídica, em Mato Grosso, MT/2003. Disponível em: <http://www.seplan.mt.gov.br/anuario2004/ 1.4.1.htm>. Acesso em: 19 set. de 2006. 
SILVEIRA, L. Ecologia e conservação dos mamíferos carnívoros do Parque

Nacional das Emas, Goiás. 1999. $117 \mathrm{f}$.

Dissertação (Mestrado em Biologia) -

Universidade Federal de Goiás, Goiânia, 1999.
WILSON, D. E.; REEDER, D. A. (Eds.). Mammal species of the world: a taxonomic and geographic reference. 3.ed. Baltimore: The Johns Hopkins University Press, 2005. 2v. 2142p. 
